\title{
Análisis situacional y generación de un mapa estratégico para el incremento del mercado potencial en una institución prestadora de salud
}

\author{
Carmen Juliana Villamizar Jaimes* \\ Andrés Villegas Mejía**
}

Recibido: 10 de agosto de 2020

Revisado: 15 de septiembre de 2020

Aprobado: 10 de octubre de 2020

Citar como:

Villamizar Jaimes, C. J. y Villegas Mejía, A. (2021). Análisis situacional y generación de un mapa estratégico para el incremento del mercado potencial en una institución prestadora de salud. Revista CIFE, 23(38). https://doi.org/10.15332/22484914.6135

\section{Resumen}

El presente artículo analiza el caso de la institución de salud 'Profesionales de la Salud y Cía. Ltda.', con una trayectoria de más de 25 años, que se ha convertido en un socio estratégico para diferentes compañías de seguros; sin embargo, los problemas financieros que enfrenta actualmente, motivan la búsqueda de nuevos mercados. Para ello, se trabajó en el desarrollo de una estrategia de marketing en salud, con el fin de identificar a nuevos posibles usuarios de esta institución, lo que permitirá generar una propuesta de valor adecuada a las necesidades insatisfechas y reducir la dependencia de las aseguradoras. Así pues, el objetivo principal de este estudio es generar un mapa estratégico de marketing, que responda a las necesidades del entorno y pueda atraer una mayor población potencial. La propuesta de investigación, que tiene lugar en paralelo con un diagnóstico de la institución, está conformada por tres ejes temáticos, cada uno de los cuales engloba una estrategia de investigación de mercado. A través de esta propuesta, se espera establecer una segmentación de los mercados objetivo que, junto con la identificación de las brechas, pueda conducir a la creación del mapa estratégico de la institución, de este modo, a partir del plan estratégico creado, la gerencia general podrá tomar decisiones con base en evidencia.

Palabras clave: institución prestadora de salud, mapa estratégico de mercadeo, análisis situacional, evaluación del cliente, evaluación de la calidad.

Clasificación JEL: I11, M31, M21, M37.

\footnotetext{
${ }^{*}$ Universidad del Rosario, Escuela de Administración, Bogotá, Colombia.

** Universidad del Rosario, Bogotá, Colombia.
} 


\title{
Situational analysis and generation of a strategic map to increase the potential market in a health care provide institution
}

\begin{abstract}
This article analyzes the case of the health care institution 'Profesionales de la Salud y Cía Ltda.', with a trajectory of more than 25 years, which has become a strategic partner for different insurance companies. However, the financial problems it is currently facing, motivate the search for new markets. To this end, we worked on the development of a health marketing strategy, in order to identify new potential users of this institution, which will allow us to generate a value proposition adapted to unmet needs and reduce dependence on insurance companies. Thus, the main objective of this study is to generate a strategic marketing map which responds to the needs of the environment and can attract a larger potential population. The research proposal, which takes place in parallel with a diagnosis of the institution, is made up of three main focuses, each of which encompasses a market research strategy. Through this proposal, it is expected to establish a segmentation of the target markets that, together with the identification of the gaps, can lead to the creation of the institution's strategic map. By doing so, from the strategic plan created, the general management will be able to make evidence-based decisions.
\end{abstract}

Keywords: health care providers, strategic marketing map, situational analysis, customer assessment, quality assessment.

JEL Classification System: I11, M31, M21, M37.

\section{Introducción}

Profesionales de la Salud y Cía. es una institución prestadora de servicios de salud (IPS), con una trayectoria de más de 25 años en el sector de la rehabilitación. Presta servicios de terapia física, terapia ocupacional, fonoaudiología, terapias especializadas y elaboración de prótesis oculares. Actualmente, Profesionales de la Salud y Cía., así como otras IPS en el país, se enfrenta a una realidad financiera de gran preocupación, debido problemáticas como la lentitud en el flujo de recursos del sistema e insuficientes acciones del Estado para garantizarlos, así como la existencia de carteras mayores a 90 días (Molina y Vargas-Jaramillo, 2010) (Asociación Colombiana de Hospitales y Clínicas, 2018), lo que evidencia la fragilidad financiera que presentan las IPS en Colombia.

Estas problemáticas, referentes a la relación EPS-IPS, son cada vez más comunes en la institución. Actualmente la empresa en cuestión presenta una cartera por parte de las EPS que supera los 180 días. Por otra parte, al analizar la rentabilidad y los márgenes brutos de ganancia de cada uno de los servicios, se encuentra que las EPS pagan una tarifa tres veces inferior a la tarifa para pacientes particulares o pacientes de otras entidades (ARL o prepagadas). 
Profesionales de la Salud y Cía. ha sido durante años un aliado estratégico para diferentes EPS de la región, actualmente la organización tiene más de 20 convenios con EPS en Santander. Durante este tiempo, la entidad se ha caracterizado por prestar servicios de calidad orientados siempre en el paciente, lo que le ha permitido obtener a lo largo de los años renovaciones de contratos con estas entidades. Los convenios con las EPS garantizan, en cierta medida, el flujo de pacientes diario, ya que estas entidades tienen un gran número de afiliados, por lo que siempre existe demanda del servicio.

Por lo anterior, las estrategias establecidas a lo largo de los años por parte de Profesionales de la Salud y Cía. para la captación de pacientes diferentes a afiliados de EPS (Aseguradoras de Riesgos Laborales, Empresas de Medicina Prepagada y pacientes particulares), han sido muy pocas. Estas poblaciones no se encuentran claramente identificadas por la empresa, dado que pertenecen a un grupo con el que no se ha tenido el suficiente contacto, por tanto, no es claro aún de qué manera se puede llegar a captar un mayor número de estos usuarios, con el fin de generar mayores ganancias para la empresa.

Desarrollar una estrategia de mercadeo en salud permitirá conocer y entender al usuario que todavía no está caracterizado por la institución, permitiéndole a esta generar una propuesta de valor adaptada a las necesidades insatisfechas, disminuyendo de esta manera la dependencia a las EPS. Por tanto, esta investigación busca resolver la pregunta: ¿Qué estrategias de mercadeo pueden implementarse con el fin de aumentar la participación de pacientes no provenientes de EPS, en la IPS Profesionales de la Salud y promover así su sostenibilidad a largo plazo?

Este trabajo de investigación tiene como objetivo principal, generar un mapa estratégico de mercadeo que responda a las necesidades del entorno de la empresa Profesionales de la Salud y Cía., que le ayude a cumplir sus objetivos a futuro, particularmente, el de captar mayor población potencial para el consumo de sus servicios de rehabilitación. De igual forma, se plantean dos objetivos específicos: diseñar y aplicar instrumentos de medición del mercado objetivo, con el propósito de establecer las expectativas y percepciones de los clientes internos y externos de la empresa Profesionales de la Salud y Cía. Además, se realizará el análisis e interpretación de las dimensiones y brechas existentes en el mercado objetivo, con la intención de proponer mejoras al servicio que actualmente ofrece la empresa.

Este artículo busca, también, evidenciar el trabajo de investigación desarrollado, exponiendo la ruta metodológica, los resultados de investigación, una propuesta de intervención y, finalmente, las conclusiones.

\section{Propuesta metodológica}

La propuesta de investigación está compuesta por 3 diferentes ejes temáticos, cada uno de los cuales abarca una estrategia de investigación de mercado. En este apartado se presenta la 
necesidad de cada una de estas estrategias, con miras a obtener una propuesta completa, que permita dar recomendaciones a la IPS Profesionales de la Salud y Cía., a efectos de aumentar su participación en el mercado.

Figura 1. Ejes de investigación

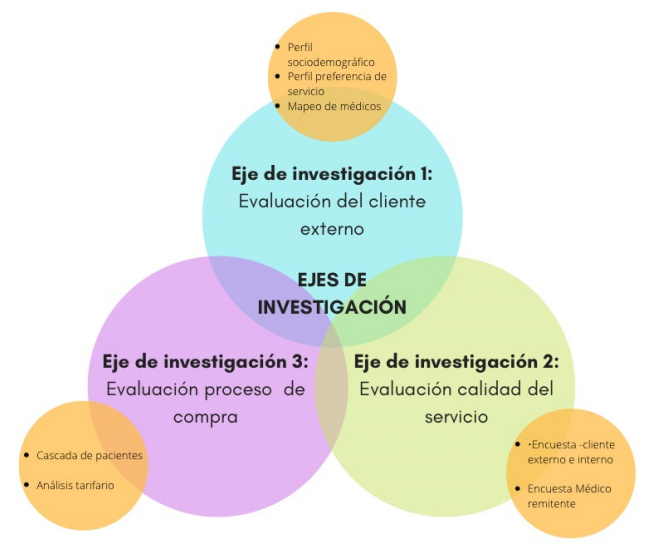

La metodología aplicada en esta investigación fue de dos tipos: descriptivo y exploratorio. Se realizó una investigación de tipo no experimental, sistemática y empírica, en donde las variables no tuvieron manipulación por parte del autor, no se realizaron intervenciones ni se tuvo influencia directa sobre las variables. Se utilizó un método de corte transversal, dado que no se realizó un seguimiento a los participantes, en cambio, se hizo recolección de datos en un solo momento. Cada uno de los ejes investigativos descritos contaron con métodos diferentes, lo que permitió nutrir de valor los resultados y conclusiones de este estudio. A continuación, se explica cada uno de ellos.

\section{Eje de investigación 1: evaluación del cliente externo}

Este eje de investigación tuvo como sujetos de análisis a cuatro tipos de cliente externo: usuarios actuales, usuarios potenciales, médicos remitentes actuales y médicos remitentes potenciales. La evaluación del comportamiento del consumidor, basado en una caracterización de la población, permite tener una mejor visión sobre la persona que asiste a servicios de salud en la institución y permite establecer estrategias centradas en el usuario y sus necesidades.

Por otra parte, se estableció la necesidad de identificar a los médicos generales y los especialistas que actualmente remiten sus pacientes al centro de rehabilitación, así como a otros médicos que pueden llegar a realizar remisiones a los servicios de la entidad, dado que la empresa no tiene actualmente una base de datos organizada donde se encuentre claramente identificada esta población. 
Figura 2. Metodología de investigación Eje 1: evaluación del cliente externo

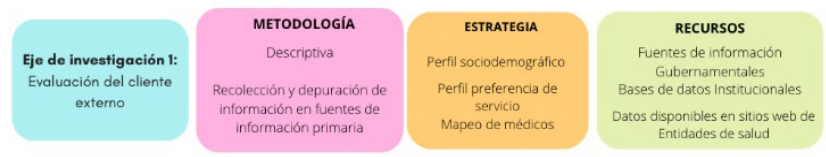

Para la recolección de información referente a la caracterización de la población afiliada del municipio, se hizo uso de fuentes de información gubernamentales como la Alcaldía de Bucaramanga y el Ministerio de Salud y Protección Social. Tanto el perfil sociodemográfico del cliente externo actual de la institución como el perfil de preferencia del servicio, se hicieron mediante la depuración de la base de datos de la empresa. Las variables tenidas en cuenta para construir con ambos un perfil único fueron: número de personas atendidas, sexo, ciclo de vida en el que se encuentran, patologías de mayor prevalencia, horario de atención preferido, porcentaje de inasistencia y estrato socioeconómico.

Por otra parte, la caracterización de la población de médicos actuales se hizo únicamente mediante la depuración de la base de datos de la empresa. Se determinaron aquellos médicos que remiten pacientes a la entidad en mayor volumen, el número total de remisiones realizadas en el año y las patologías presentadas con mayor frecuencia. Se hace la caracterización en las 5 entidades por medio de las cuales se realiza la mayoría de las atenciones.

La metodología de búsqueda de médicos potenciales implicó la revisión de directorios telefónicos disponibles en los sitios web de las entidades de medicina prepagada más reconocidas en la ciudad, con las que actualmente se tiene convenio y que tenían en sus páginas el directorio médico de libre consulta. Se realizó, entonces, un mapeo por especialidades, tomando en cuanta cuáles pacientes podrían ser remitidos para procesos de rehabilitación.

\section{Eje de investigación 2: evaluación de la calidad del servicio}

Se hace necesario en este estudio evaluar la percepción que tienen los usuarios, médicos remitentes y profesionales de la IPS Profesionales de la Salud y Cía. Ltda., sobre la calidad en los servicios de rehabilitación.

Entre los principales modelos de evaluación de la calidad de los servicios de salud, desde la percepción del usuario, se destaca el instrumento SERVQUAL (Service Quality). Este modelo fue validado en Latinoamérica por primera vez en 1992 y desde entonces ha sido ampliamente usado y validado en países como España, Colombia e Irán (Numpaque-Pacabaque y Rocha-Buelvas, 2016). Para SERVQUAL, la calidad es el resultado de la diferencia entre las expectativas y las percepciones de un servicio (Pérez et al., 2018; Yepes et al., 2018; Matsumoto, 2014; Cabello y Chirinos, 2012). A continuación, 
se describen los instrumentos utilizados, la muestra poblacional seleccionada y, finalmente, la metodología usada para el análisis de datos.

Figura 3. Metodología de investigación Eje 2: evaluación de la calidad del servicio
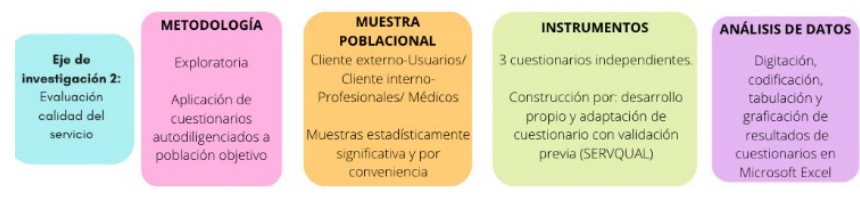

\section{Instrumentos}

La aplicación de los diferentes cuestionarios se realizó de manera secuencial. En un primer momento se recolectó la muestra de la población de usuarios por medio del cuestionario 'Cliente externo', en un segundo momento se recolectó la muestra de la población médicos, por medio del cuestionario 'Médicos' y, finalmente, la muestra de profesionales, por medio del cuestionario 'Cliente interno'. Lo anterior, dado que este último cuestionario fue adaptado de acuerdo con lo hallado en los primeros dos momentos; de modo que las preguntas seleccionadas para el cuestionario de profesionales se escogieron luego de establecer las variables en las cuales se quería profundizar y contrastar entre las tres poblaciones.

Cuestionario cliente externo: el cuestionario de cliente externo tiene como objeto, en primer lugar, caracterizar una muestra representativa de la población que asiste a servicios de rehabilitación en la entidad $\mathrm{y}$, en segundo lugar, evaluar la percepción de la calidad del servicio en la entidad. La primera parte contó con un cuestionario de 11 preguntas abiertas, desarrollo propio de la investigación.

Para la segunda parte del cuestionario se hizo una adaptación del cuestionario SERVQUAL. Se mantuvo la misma cantidad de ítems y se conservó su división por dimensiones, sin embargo, se hizo un ajuste en el contenido de aquellos, con efecto de que estos fueran específicos para servicios de rehabilitación. Esta segunda parte constó de 22 ítems contenidos en 5 grandes dimensiones: tangibilidad, empatía, responsabilidad, capacidad de respuesta y fiabilidad. Estos 22 ítems fueron evaluados en dos momentos diferentes (a través de las respuestas de un total de 44 preguntas), mediante una puntuación con escala tipo Likert. En el caso de la importancia de los factores en un servicio de rehabilitación, la escala utilizada fue: Nada importante; Poco importante; Neutral; Importante; Muy importante.

Por otra parte, la escala utilizada para evaluar la percepción dentro de la institución fue: Mucho menor de lo esperado; Menor de lo esperado; Lo que esperaba; Mayor de lo esperado; Mucho mayor de lo esperado (Calixto-Olalde et al., 2011; Prieto et al., 2011). Así, en una primera parte se indagó sobre el grado de importancia que el cliente asigna a los diferentes ítems para garantizar una satisfacción total en un servicio de rehabilitación. De otro lado, en la segunda parte se indagó sobre cómo fueron observados esos ítems según su reflejo en el 
servicio brindado por la entidad. Esto, con el propósito de comparar la expectativa frente a lo obtenido en la entidad.

Cuestionario a médicos: el cuestionario buscaba caracterizar las remisiones realizadas por médicos a servicios de rehabilitación (el tipo de pacientes atendidos, sesiones ordenadas, importancia percibida sobre rehabilitación) e identificar la percepción de calidad del servicio brindado en profesionales de la salud. Este cuestionario fue un desarrollo propio de la investigación, dado que no se encontró en la literatura un instrumento que permitiese responder este tipo de preguntas.

La primera parte del cuestionario, en la que se tenía como objetivo indagar sobre datos generales del médico, utilizó preguntas con respuesta abierta. La segunda parte consistió en 10 preguntas, las cuales fueron estructuradas con respuestas múltiples o de escala. Esto, con el objeto de facilitar el análisis de los datos obtenidos.

Cuestionario cliente interno: por medio de este cuestionario se buscó evaluar la percepción de la calidad del servicio prestado por la entidad y caracterizar las remisiones que médicos generales y especialistas realizan a esta, todo ello desde la visión del rehabilitador. Para lograr esto se utilizaron apartados tanto del cuestionario realizado a usuarios cómo del cuestionario realizado a médicos. Lo anterior, con el objetivo de llevar a cabo la triangulación de información, nutriendo de esta manera los resultados y las posibles estrategias que pudieran llegarse a proponer.

Se utilizó la misma escala de valoración de los 22 ítems (con sus respectivas preguntas espejo), pero esta vez aplicada a usuarios. Además, se utilizaron 5 de las preguntas del cuestionario de médicos, referentes a tipos y números de referencia a los servicios (ver Anexo 5). Por tanto, para la creación de estos cuestionarios se hizo uso de los métodos con los cuales se desarrollaron los dos cuestionarios anteriores, es decir, se basaron tanto en un desarrollo propio como en la adaptación del cuestionario SERVQUAL.

\section{Muestra poblacional}

Población de usuarios: usuarios que en el momento de la aplicación de la encuesta estén asistiendo a servicios de rehabilitación en la IPS. Para establecer una muestra estadísticamente significativa se realizó el siguiente cálculo del tamaño muestral, teniendo como intervalo de confianza del $95 \%$ :

$$
n=\frac{Z^{2} * P * q * N}{\left(E^{2} * N\right)+\left(Z^{2} * P * q\right)}
$$

Donde:

n: tamaño de la muestra. 
$\mathrm{N}$ : tamaño de la población: 1000 (correspondiente al número promedio de usuarios en el servicio de consulta externa en consultorio en la entidad).

Z: nivel de confianza deseado: 1,96 equivalente al $95 \%$ de fiabilidad.

P: factor de aceptación de la muestra: $50 \%(0,50)$.

q: factor de rechazo de la muestra: $50 \%(0,50)$.

E: porcentaje de error admisible para la muestra: $5 \%(0,05)$.

Los valores establecidos fueron seleccionados teniendo en cuenta las características del presente estudio y de estudios anteriores de calidad en servicios de salud (Acevedo, 2015).

$$
\begin{gathered}
n=\frac{1,96^{2} * 0,5 * 0,5 * 3000}{\left(0,05^{2} * 1000\right)+\left(1,96^{2} * 0,5 * 0,5\right)} \\
n=248
\end{gathered}
$$

Población de profesionales: profesionales en rehabilitación (fisioterapeutas, terapeutas ocupacionales o fonoaudiólogos) que en el momento de la aplicación de la encuesta laboren en la IPS. Se establecieron los mismos parámetros estadísticos que en la muestra anterior. Sin embargo, en esta el tamaño de la población corresponde a 60 (profesionales en rehabilitación por nómina en la entidad).

$$
\begin{gathered}
n=\frac{1,96^{2} * 0,5 * 0,5 * 80}{\left(0,05^{2} * 80\right)+\left(1,96^{2} * 0,5 * 0,5\right)} \\
n=52
\end{gathered}
$$

Población de médicos: médicos generales y especialistas con historia de remisión de pacientes a la entidad. El criterio para la escogencia de la muestra estaba relacionado con la conveniencia; se estableció una meta de recolección de 25 cuestionarios.

\section{Recolección de la muestra}

La muestra de usuarios fue recolectada en un periodo de dos semanas, por un grupo de encuestadores capacitados, los cuales escogieron de manera aleatoria usuarios en salas de espera y consultorios. De igual modo, la muestra de profesionales se realizó de manera aleatoria durante su jornada laboral; el cuestionario fue autodiligenciado y contó con presencia del evaluador al momento de su aplicación. Finalmente, el cuestionario a médicos se realizó de manera telefónica o mediante el envío del cuestionario vía correo electrónico.

\section{Análisis de los datos}

Los procesos de digitación, codificación, tabulación y graficación de los resultados obtenidos en las encuestas se realizó en el programa Microsoft Excel. Las preguntas abiertas en el 
primer apartado del cuestionario a cliente externo requirieron un proceso de codificación mediante la metodología de categorías emergentes. Las respuestas obtenidas en cada una de las preguntas fueron enlistadas en una matriz y a cada una de ellas se le fue asignado un código, para permitir la tabulación adecuada de datos en Microsoft Excel. Por otra parte, los resultados obtenidos con base en las preguntas con respuesta tipo Likert fueron, en los tres cuestionarios, codificados con números de 1 a 5 , siendo 5 el valor de mayor importancia. Finalmente, aquellas respuestas donde el resultado correspondió a un número fueron digitadas sin cambio alguno.

\section{Eje de investigación 3: evaluación del proceso de venta}

En la figura 4 se muestra la metodología de investigación llevada a cabo en el Eje 3.

Figura 4. Metodología de investigación Eje 3: evaluación del proceso de venta
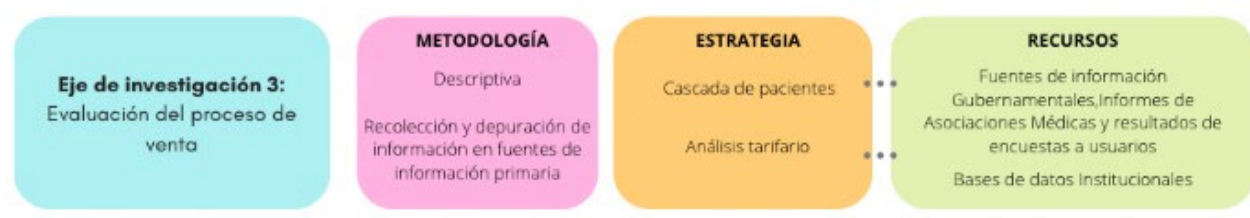

Mediante los sistemas de información de la organización se puede establecer la cantidad de pacientes que asisten a la entidad, sin embargo, este instrumento no permite identificar la cantidad de pacientes de cada una de estas entidades que podrían ser potenciales usuarios.

El Patient Flow Analysis o 'Cascada de pacientes', es una técnica de administración que permite encontrar alternativas para aumentar la eficiencia de los servicios. Además, posibilita la identificación de vacíos en la distribución y utilización de los servicios, evaluando la manera como los pacientes transitan a través del servicio (Assefzadeh, 1996; Dixon et al., 2015), a efectos de establecer áreas de intervención que permitan aumentar la participación de pacientes no provenientes de EPS en la IPS Profesionales de la Salud.

De igual manera, dentro de la evaluación del proceso de compra se hace necesario analizar las tarifas pactadas entre la IPS y las diferentes entidades de salud, para poder establecer propuestas de mejora en el área.

La estructuración de la cascada de pacientes se realizó a partir de cinco eslabones, donde para cada uno ellos se contó con fuentes de información diversas. Para los primeros dos eslabones, correspondientes a población total del municipio y población afiliada por regímenes, se utilizaron fuentes primarias gubernamentales, tales como: Supersalud y Ministerio de Salud y Protección Social. Asimismo, se realizó una depuración de datos de los informes: el 'Informe de población afiliada a las Empresas de Medicina Prepagada y Planes Complementarios' y la 'Caracterización de la población afiliada a las Entidades Administradoras de Planes de Beneficios'. 
Para el desarrollo del tercer eslabón se hizo necesario encontrar un indicador que permitiese establecer la manera como los usuarios asisten a servicios de rehabilitación. La extensión de uso indica el porcentaje de personas afiliadas que utilizaron servicios de salud en un periodo específico (Asociación Colombiana de Empresas de Medicina Integral, 2017). El valor utilizado como extensión de uso fue de $71 \%$ (Asociación Colombiana de Empresas de Medicina Integral, 2017).

Los datos del cuarto eslabón de la cascada de pacientes fueron obtenidos a partir del sistema de información de Profesionales de la Salud y Cía. y correspondieron al número de pacientes atendidos en la entidad durante el año 2019. Finalmente, para el último eslabón de la cascada se hizo uso de información procedente del 'Cuestionario a cliente externo', específicamente de la pregunta: “¿Ha asistido anteriormente a la entidad?”. Teniendo en cuenta el significado estadístico que presenta este cuestionario, y dada la cantidad de personas a las cuales fue aplicado, se hace uso de este porcentaje para estimar la posible reutilización de los servicios de rehabilitación de la entidad. Este quinto eslabón sirve para caracterizar el porcentaje de pacientes que vuelven a los servicios de rehabilitación en la IPS Profesionales de la Salud y Cía.

En cuanto a la metodología usada para el análisis tarifario, se hizo una revisión del documento institucional "Manual tarifario", en el cual se encuentran establecidos cada uno de los valores pactados con las diferentes entidades de salud para los servicios prestados por la institución; datos que fueron depurados y organizados en diferentes tablas. Finalmente, se utilizó una estrategia de semaforización para comparar y analizar información confidencial de la empresa, con el fin de generar recomendaciones para optimizar su desempeño.

\section{Resultados de investigación}

Los resultados obtenidos en cada uno de los ejes de investigación permitieron recolectar información valiosa para la evaluación del proceso de decisión de compra del servicio; de igual forma, se identificaron las brechas en el sistema y se logró establecer el mercado potencial de la institución. Finalmente, con estos resultados analizados, se estableció una matriz producto del estudio de todo el documento presentado y se realizó una evaluación mediante el análisis DOFA, el cual permite distinguir claramente las estrategias a realizar, con el propósito de ser planteadas mediante un mapa estratégico.

\section{Segmentación del mercado objetivo}

El proceso de segmentación del mercado objetivo se realiza a partir del análisis de las características propias de los individuos. Esto, con el ánimo de lograr un posicionamiento específico en cada uno de los segmentos. Existen tres condiciones que permiten realizar una adecuada segmentación del mercado: 1) el segmento debe ser accesible por los canales actuales; 2) debe suponer un tamaño suficientemente grande; y 3) su distinción con otros 
grupos debe ser claramente medible. Por otra parte, el proceso de segmentación supone tres pasos: 1) la identificación de las necesidades insatisfechas, satisfechas y no reconocidas; 2) la identificación de características diferenciadoras de otros grupos; y 3) el cálculo de la demanda actual y potencial de cada segmento.

Figura 5. Análisis de resultados

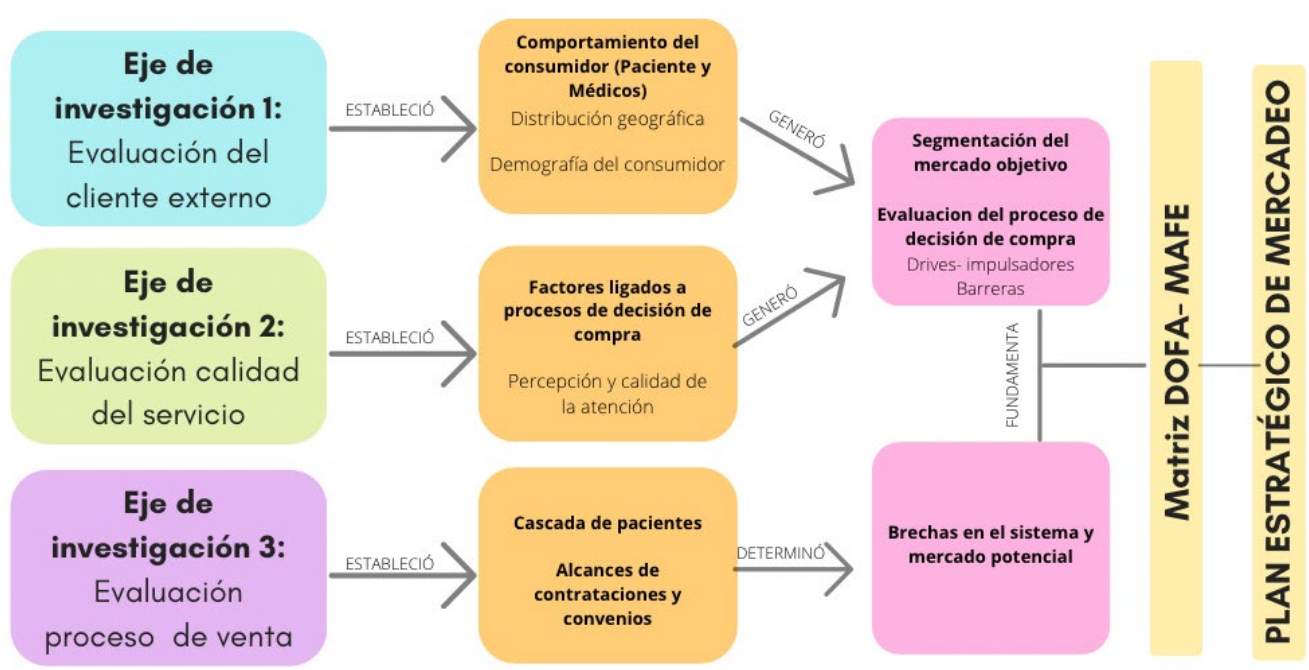

Teniendo en cuenta el proceso de segmentación anteriormente descrito, se realizó una evaluación de las variables sociodemográficas del cliente actual, las cuales permitieron generar un perfil del usuario. Realizar una segmentación por características sociodemográficas en este contexto resulta de gran importancia, dado que estas características demarcan claramente los patrones de consumo del usuario. Las necesidades en salud están profundamente ligadas a las variables sociodemográficas, por tanto, la elaboración de este perfil puso en evidencia diferencias claves entre los usuarios, que fomentarán la posible ampliación de servicios que actualmente ofrece la institución.

El primer segmento clave identificado mediante la ‘Caracterización del cliente interno’ (Eje de investigación 1) fue la población de sexo femenino, la cual representa más del $60 \%$ de los usuarios que asisten actualmente a la institución. De igual forma, esta proporción también se evidenció en el 'Cuestionario a cliente externo' (Eje de investigación 2), en donde el 62 \% de la población encuestada correspondió al sexo femenino. Finalmente, en la 'Caracterización de la población bumanguesa' (Eje de investigación 1) se encontró también una mayor proporción de personas de sexo femenino. Estos tres datos ratifican la importancia de esta población dentro del análisis y, por tanto, la necesidad de establecer estrategias guiadas específicamente para ella.

El segundo segmento poblacional clave identificado fue la población correspondiente a los adultos mayores. Esta representa una alta proporción en el municipio (79 000 personas), adicionalmente, el índice de envejecimiento en la ciudad se duplicó desde su última medición. 
Por otra parte, en la 'Caracterización del cliente interno' se encontró que el grupo poblacional que más asiste a servicios de rehabilitación está conformado por adultos mayores (más de 14000 usuarios al año). De igual manera, en el 'Cuestionario a cliente externo' se encontró una media poblacional por encima de 45 años, lo que ratifica la importancia de este segmento poblacional en el análisis y la necesidad de generar estrategias específicas.

El tercer segmento poblacional clave identificado fue el de pacientes con patologías de origen osteomuscular; y el quinto correspondió al de pacientes con patologías crónicas. Este se pudo identificar a partir de la 'Caracterización de la población afiliada a régimen contributivo en Bucaramanga,' la 'Caracterización de cliente interno' a partir del sistema de información de la empresa, y, finalmente, a la pregunta 'Patologías mayormente referidas a servicios de rehabilitación', incluida en los cuestionarios realizados a cliente interno y médicos. Información fundamental, pues los pacientes con patologías crónicas reincidirán en los servicios de rehabilitación.

Finalmente, las patologías osteomusculares evidenciaron ser un segmento importante, pues requieren de un gran trabajo de rehabilitación. Esto, a partir de los análisis de caracterización realizados en el Eje de investigación 1 y lo referenciado tanto por profesionales como por médicos en los cuestionarios (Eje de investigación 2).

La empresa cuenta con equipos de última tecnología especializados en manejo de dolor en patologías crónicas; son pocos los centros de rehabilitación en el país que actualmente cuentan con esta tecnología. Sin embargo, son poco conocidos tanto por los usuarios como por la mayoría de terapeutas de la institución (esto se pudo evidenciar mediante el ítem 'Equipos modernos', evaluado en el cuestionario de cliente interno y externo; ítem que tuvo una puntuación relativamente baja en ambos cuestionarios). Lo anterior se debe a que son servicios premium que pocos profesionales están capacitados para manejar; además, son también pocos los pacientes que llegan a solicitar tales servicios debido a su costo; finalmente, el mercadeo de estos, tanto a nivel interno como externo, ha sido mínimo.

De igual manera, establecer estos grupos clave de intervención permite entender la demanda del mercado, y, por tanto, guiar la compra de equipos y la formación del profesional que realizará la atención de estas poblaciones.

\section{Proceso de compra}

El proceso de decisión de compra consta de 5 fases: 1) reconocimiento de la necesidad; 2) búsqueda de la información; 3) evaluación de las alternativas; 4) decisión de compra; y 5) comportamiento posterior a la compra. Los resultados obtenidos en los ejes de investigación 1 y 2 permitieron establecer elementos claves en este proceso, tales como impulsores y barreras. Tanto las EPS como los médicos o los profesionales pueden convertirse en barreras 
o impulsores para el acceso al servicio. Por lo anterior, la visión de estos actores cobra relevancia durante el análisis del proceso de compra.

Figura 6. Proceso de compra
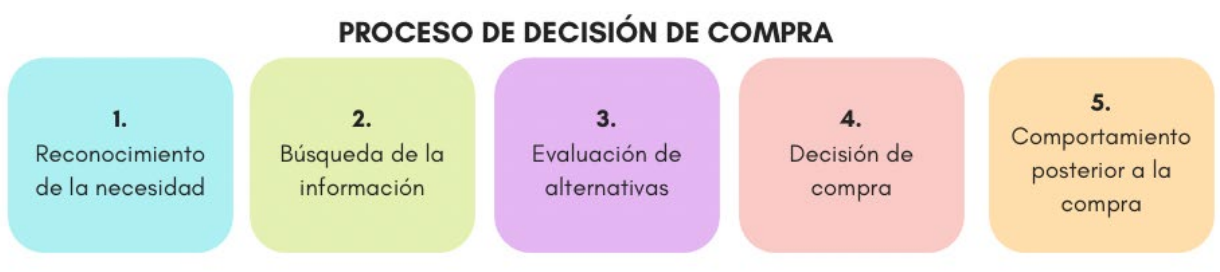

\section{Reconocimiento de la necesidad}

El proceso de compra inicia con el reconocimiento de la necesidad. El usuario tiene dos formas de llegar a los servicios de rehabilitación: de manera particular o mediante la remisión de un médico general o especialista. Cuando los procesos patológicos tienen implicaciones estructurales, funcionales o restrictivas en la participación, es fácil para el usuario darse cuenta de que algo está mal, por tanto, el reconocimiento de la necesidad es claro. Sin embargo, cuando son procesos silenciosos, como en las enfermedades crónicas no transmisibles, el reconocimiento de la necesidad no es claro.

Mediante los cuestionarios realizadas a médicos y profesionales, se encontró que estos asignaron una baja puntuación al tratamiento de enfermedades crónicas no transmisibles por parte de servicios de rehabilitación. Sin embargo, en los nuevos modelos de atención en salud, guiados hacia la prevención, estas intervenciones serán una pieza fundamental en los abordajes, por tanto, crear un mayor reconocimiento de estas necesidades en los pacientes y la comunidad del sector salud permitirá aumentar la compra de servicios.

\section{Búsqueda de información}

El segundo paso del proceso de decisión de compra es la búsqueda de información. Mediante los tres cuestionarios realizados en el segundo Eje de investigación, se hallaron resultados que pueden afectar esta fase del proceso. Se encontró, por ejemplo, que los médicos no conocen la totalidad de los servicios ofrecidos por la institución; los profesionales de la institución tampoco conocen la oferta de servicios completa que la institución ofrece; y, finalmente, ninguno de los usuarios entrevistados mencionó haber conocido el servicio por medio de internet (un canal apto para la búsqueda de información sobre la institución). Por lo anterior, se concluye que es necesario empoderar tanto a médicos como profesionales sobre los servicios que ofrece la institución.

En concordancia con lo anterior, deben darse a conocer al interior de la organización los diferentes servicios de todas las áreas, para que sean los mismos profesionales quienes se encarguen de identificar y motivar a sus pacientes a adquirir estos servicios adicionales. Es de vital importancia capacitar al cliente interno de la empresa en temas de mercadeo, con el 
fin de que conozca la estrategia y entienda su rol protagónico en el posicionamiento de la entidad frente a sus clientes.

De igual manera, es necesario fortalecer la coordinación asistencial entre médicos y profesionales en rehabilitación, con el objeto de que puedan brindar una mayor y mejor información a los usuarios, que les permita aumentar la intensidad del estímulo para asistir a servicios de rehabilitación. Se observó en las encuestas, tanto a médicos como a profesionales, que el número promedio de citas está por encima de 15. Este puede ser un tiempo alto para algunas de las patologías, las cuales pueden ser resueltas más rápidamente, según las guías internacionales de manejo. Por tanto, realizar consensos entre las partes sobre la cantidad de citas permitirá: 1) mejorar la oportunidad de las citas, dado que se liberará agenda con mayor rapidez; 2) aumentar la eficiencia de las intervenciones y los márgenes en los contratos por capitación; y 3) que los pacientes vean una resolución de su cuadro clínico en menor tiempo.

\section{Evaluación de las alternativas}

El tercer paso en el proceso de decisión de compra es la evaluación de alternativas. Los cuestionarios realizados tanto a cliente interno, externo y médicos mostraron muy buenas percepciones respecto a la calidad del tratamiento, el trato humanizado y el conocimiento del profesional. Estos, sin duda, son los valores por los cuales es reconocida la institución, por ello, estas características deben consolidarse como la ventaja competitiva de la institución en el exterior. Las campañas publicitarias a realizar deben enfatizar estos aspectos, dado que son factores ampliamente reconocidos por los diferentes actores; lo anterior, en aras de que el usuario, al evaluar las diferentes alternativas en el mercado, vea un factor diferencial en esta institución y sea un driver para realizar la compra del servicio.

Las encuestas realizadas a cliente interno y a cliente externo evidenciaron descuido en los temas relacionados con la publicitad de la institución, por tanto, es necesario crear un plan estructurado de comunicación, especialmente en medios digitales. Esto, tomando en cuenta que la gran cantidad de usuarios en edades jóvenes o adultas, pues los procesos de compra de estas poblaciones están alimentados por lo encontrado en redes sociales e internet. Por tanto, el marketing digital debe cobrar mayor relevancia en el plan estratégico de la institución, con el fin de brindar al usuario más elementos para que pueda evaluar las alternativas de servicio.

\section{Decisión de compra}

Una vez el cliente ha hecho la evaluación de las alternativas, realiza la decisión de compra, la cual constituye el cuarto paso dentro del proceso de compra. Dentro de esta etapa, el consumidor toma en cuenta sus preferencias con respecto al servicio y decide con base en diferentes varibles: decisión de marca, vendedor, cantidad, tiempo, entre otras. Es muy importante brindar al usuario una ámplia gama de alternativas, pues más del $95 \%$ de los 
usuarios actuales llegan al servicio debido a una remisión directa de su EPS, lo que significa que no tienen posibilidad de escoger la institución a la cual pueden asistir para su rehabilitación. Es necesario, entonces, establecer puntos de oportunidad donde el usuario pueda llegar a personalizar su servicio y sentir que es tenido en cuenta para diferentes decisiones involucradas en su proceso de tratamiento.

Ofrecer diferentes opciones al usuario aumentará en él la satisfacción respecto del servicio. Entre las posibles variables que pueden ser fácilmente tenidas en cuenta para este fin están: permitir escoger el lugar de la atención, es decir, brindar la posibilidad al usuario de escoger en cuál de los diferentes centros que tiene la institución quiere ser atendido y en qué horario. Para esta variable se encontró, según el horario de preferencia para la utilización del servicio, que los horarios más usados son los horarios matutinos, por tanto, reforzar este turno con personal de medio tiempo permitiría aumentar la capacidad instalada en horas de alta demanda. Esto aumentaría la productividad de algunos trabajadores que presentan horarios con poca ocupación. Además, se debe evaluar la posibilidad de eliminar los turnos cercanos a mediodía y aumentar los horarios a primeras horas de la mañana o finalizando la jornada. Esto permitiría a usuarios que trabajan y no tengan permiso para ausentarse, poder asistir a rehabilitación en horarios no laborales. Finalmente, tener puntos de atención en zonas de alta demanda permite disminuir gastos a la población, lo que disminuye significativamente las barreras de acceso al servicio. Todo lo anterior influye positivamente en la decisión de compra de los servicios, lo que termina por aumentar la utilización de los mismos a través del tiempo, generando mayores ingresos a la institución.

\section{Comportamiento posterior a la compra}

El quinto y último paso dentro del proceso de compra se da una vez el paciente decide realizar su proceso de rehabilitación en la institución, se trata del comportamiento posterior a la compra. Esta fase es igual o más importante que las anteriores, dado que garantizará recompra de servicios. Aquí se evalúan las expectativas en contraste con el desempeño del servicio.

La segunda parte del cuestionario realizado al cliente externo permitió establecer esta comparación. Así, 20 de los 22 factores evaluados por los usuarios fueron señalados como: "según lo esperado" o "mejor de lo esperado", por tanto, se concluye que cumple con las expectativas del usuario influyendo positivamente en la recompra del servicio.

De igual manera, en esta recompra de servicios se encontraron datos positivos valiosos para el análisis. La mayor parte de los médicos encuestados refirió haber remitido pacientes por más de 10 años a la institución, esto, además de ser un voto de confianza por parte de los médicos, es una prueba de la satisfacción con el servicio. Adicional a ello, más del $40 \%$ de los pacientes encuestados retornó a los servicios. 
Los cuestionarios a cliente interno y externo pusieron en evidencia una carencia en el seguimiento al tratamiento una vez finalizadas las sesiones. En el cuestionario a cliente interno se encontró que el ítem 'Seguimiento una vez se concluye la intervención' tuvo una media de calificación que se ubicó en 'menor de lo esperado'. De igual forma, en el cuestionario a cliente externo, la categoría 'fiabilidad', de la cual hace parte el aspecto 'Seguimiento una vez se concluye la intervención' tuvo la segunda puntuación más baja.

Garantizar la continuidad en el servicio posventa es crucial para generar en el cliente una buena referencia de los servicios, y para ser considerado como primera opción en un evento futuro. Por tanto, hacer un seguimiento telefónico de los usuarios para evaluar la atención recibida, contemplar la posibilidad de ampliar sus sesiones de rehabilitación o hacer mercadeo de nuevos servicios, son acciones que podrían fomentar que la institución esté en el top of mind de los usuarios.

El análisis de los resultados a través del proceso de decisión de compra del usuario permite establecer una propuesta estratégica de mercadeo fundamentada en la evidencia. Por tal razón, y con el propósito de hacer más robustas las bases de esta propuesta, se analizarán por los resultados obtenidos en el Eje de investigación 3: evaluación del proceso de venta.

\section{Brechas en la utilización del servicio}

En este eje se evidenciarion 3 brechas en la utilización de los servicios de rehabilitación, como resultado del análisis de la cascada de pacientes. La primera brecha identificada se dio entre la población afiliada y la población que usa servicios de rehabiltiación. Para la disminución de esta brecha es necesario aumentar la extensión de uso de los servicios, esto se puede lograr mediante el fomento de actividades de rehabiltiación encamindas a la prevención de enfermedades y promoción de la salud. Desde el servicio de rehabilitación, es posible la construcción de estrategias, planes y programas que promuevan estilos de vida saludables. Estos programas podrán ser ofrecidos a las diferentes entidades con las que actualmente se tienen convenios como un servicio costo-efectivo, debido a que disminuirá las enfermedades crónicas no transmisibles en los afiliados, reduciendo así la extensión y frecuencia de uso de servicios de alto costo.

La segunda brecha identificada fue la de mayor volumen, esta se da entre los pacientes que deben asistir a servicios de rehabilitación y los que finalmente terminan asistiendo a la institución. A este respecto, se encontraron varias similitudes entre las entidades reportadas en el cuestionario a médicos como las más conocidas por estos y aquellas con las que Profesionales de la Salud y Cía. tienen contratación. Esto significa que uno de los puntos a mejorar está relacionado con el conocimiento que tienen los médicos sobre las entidades con las cuales Profesionales de la Salud tiene convenio, pues, al no conocer esto, no refieren a los pacientes a la institución, lo que puede ser una de las causantes de esta brecha. 
En este orden de ideas, el mapeo de médicos realizado en el Eje de investigación 1 cobra relevancia como una de las estrategias para manejar esta brecha, la cual disminuiría al conocer y establecer relaciones con los médicos actuales y potenciales. Como se mencionó anteriormente en este capítulo, es necesario afianzar las relaciones interinstitucionales que tiene la empresa, con el fin de direccionar a los pacientes que requieren de servicios de rehabilitación hacia Profesionales de la Salud y Cía.

Por otro lado, todas las estrategias publicitarias que permitan aumentar el conocimiento del usuario sobre el servicio repercutirán en la reducción de esta brecha. Finalmente, como se evidenció en el Eje de investigación 3, con respecto al análisis tarifario, existen entidades con las cuales actualmente se tienen convenios y que cuentan con tarifas que resultan favorables en comparación con otras del mercado; sin embargo, su representación en la cascada de pacientes es poca. Por tanto, para disminuir esta brecha y generar mayores ingresos a la institución por actividad, es necesario fortalecer los convenios con entidades de medicina prepagada.

La tercera brecha identificada está relacionada con la recompra de servicios, lo que guarda estrecha relación con el quinto paso del proceso de decisión de compra, es decir, el comportamiento posterior a la compra. El análisis planteado en este apartado permitirá establecer las estrategias que generarán una disminución en esta última brecha.

Concluido el análisis de los resultados, habiendo identificado los factores que promueven y limitan la compra de servicios de rehabiltiación y las brechas para el uso de los servicios, es posible generar una propuesta de mapa estratégico que permita a la institución aumentar su mercado potencial.

\section{Análisis estratégico}

El análisis de la organización y de los tres ejes de investigación del presente estudio permiten el desarrollo de un diagnóstico estratégico. Así las cosas, tomando ciertos elementos del análisis de las fuerzas del mercado establecido por Porter (2008), se presenta a continuación una conclusión con respecto a los siguientes aspectos: evaluación del entorno, evaluación de la competencia, evaluación de la cultura corporativa, y evaluación del perfil competitivo interno. 
Figura 7. Diagnóstico estratégico
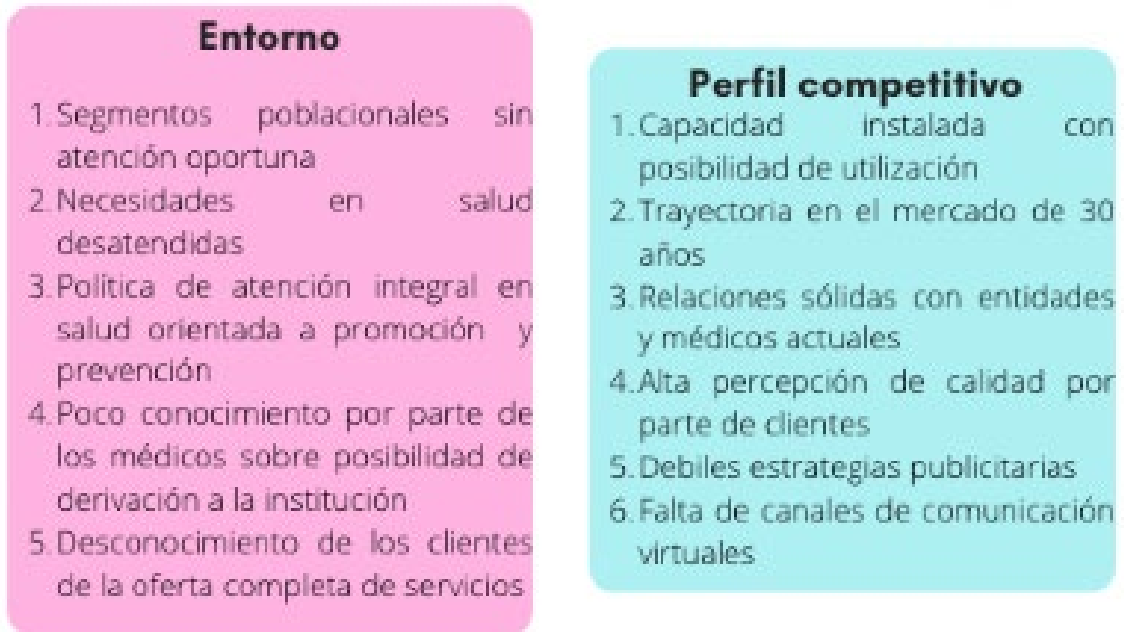

\section{DIAGNÓSTICO ESTRATÉGICO}

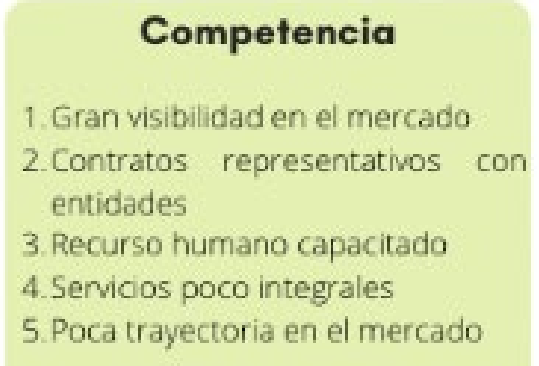

Competencia

1. Gran visibilidad en el mercado

Contratos representativos con

entidades

4. Servicios poco integrales

5. Poca trayectoria en el mercado

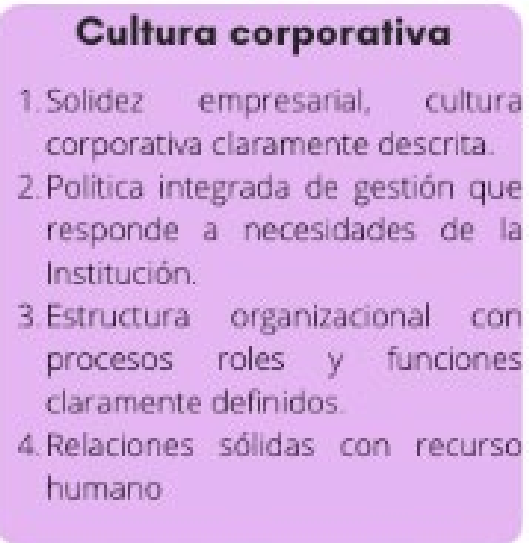

La matriz DOFA se constituye como una herramienta que permite la obtención de una perspectiva global de la situación estratégica de la institución. Este instrumento permite condensar lo encontrado en cada uno de los ejes de investigación, en el diagnóstico estratégico y en el análisis de resultados, lo que permite establecer las estrategias claves a seguir en el mapa estratégico (Brandenburger, 2019; Thompson y Strikland, 1998). Se establecen, entonces, las fortalezas y debilidades propias de la institución y las amenazas y oportunidades encontradas en el entorno.

Una vez se efectúa la matriz DOFA es posible construir la matriz MAFE. La figura 8 muestra las estrategias que responden directamente al análisis realizado a través de todo el trabajo de investigación, las cuales se encuentran alineadas con los objetivos de la organización y su ideal de mejora continua. Cabe recordar que cada una de las estrategias toma como base uno o varios segmentos de la matriz DOFA. 
Figura 8. Matriz MAFE
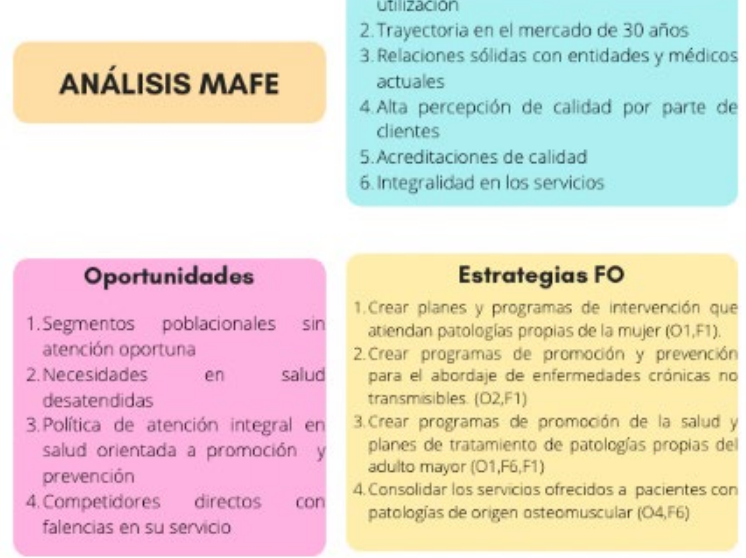

Amenazas

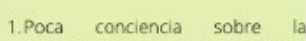

importancia de la promoción y prevención

2. Poco conocimiento por parte de los médicos sobre posibilidad de derivación a la institución

3. Desconocimiento de los clientes

de la oferta completa de servicios

4. Tarifas en el mercado bajas

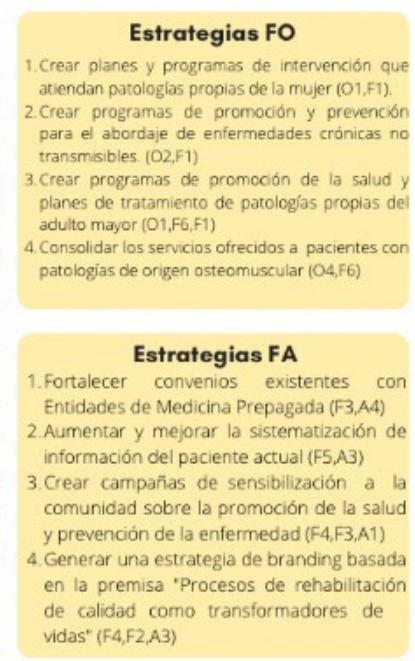

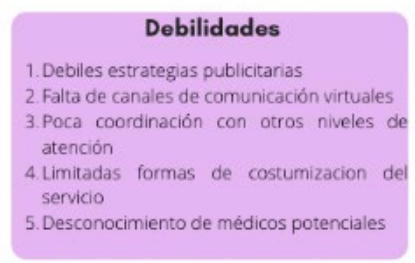

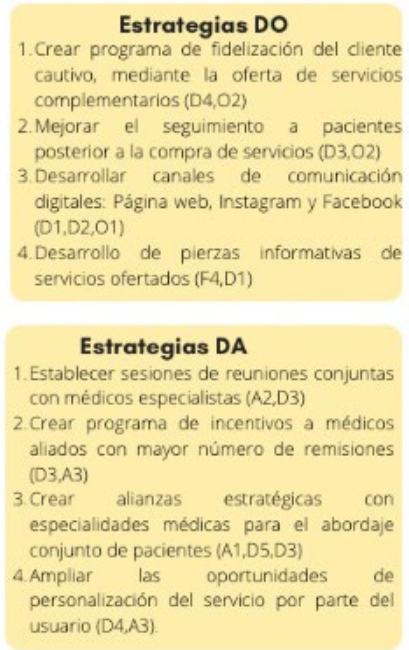

\section{Propuesta de intervención-mapa estratégico de mercadeo}

El entendimiento profundo de la situación actual de la organización mediante el desarrollo de los 3 ejes de investigación - teniendo como objetivo central la captación de mayor población potencial para el consumo de servicios - y el análisis mediante las herramientas DOFA y MAFE, permiten la generación de un mapa estratégico de mercadeo el cual ayudará a la institución a alcanzar su visión para el año 2021: "Para 2021, Profesionales de la Salud y Cía. espera ser la empresa líder en el departamento de Santander en la prestación de servicios de rehabilitación especializada, con un alto nivel de satisfacción de sus usuarios". De igual forma, este mapa contribuirá a la obtención de la certificación NTC 5801 (Icontec, 2008), dado que esta tiene entre sus requisitos la realización de una evaluación de su mercado actual.

Esta hoja de ruta delimita y prioriza el foco de intervención de la institución, mediante el establecimiento de objetivos enmarcados en cuatro grandes perspectivas, cada una las cuales se desarrolla a partir de un objetivo general que, a su vez, tiene cuatro objetivos específicos (figura 9), cada uno de ellos enfocado en atender una necesidad evidenciada en el análisis de resultados. De igual manera, se establecen indicadores de resultado, basados en la información recopilada, y se proponen las diferentes áreas que pueden llegar a desarrollar cada uno de estos. A continuación, se explica el mapa en detalle. 
Figura 9. Mapa estratégico 2020-2021

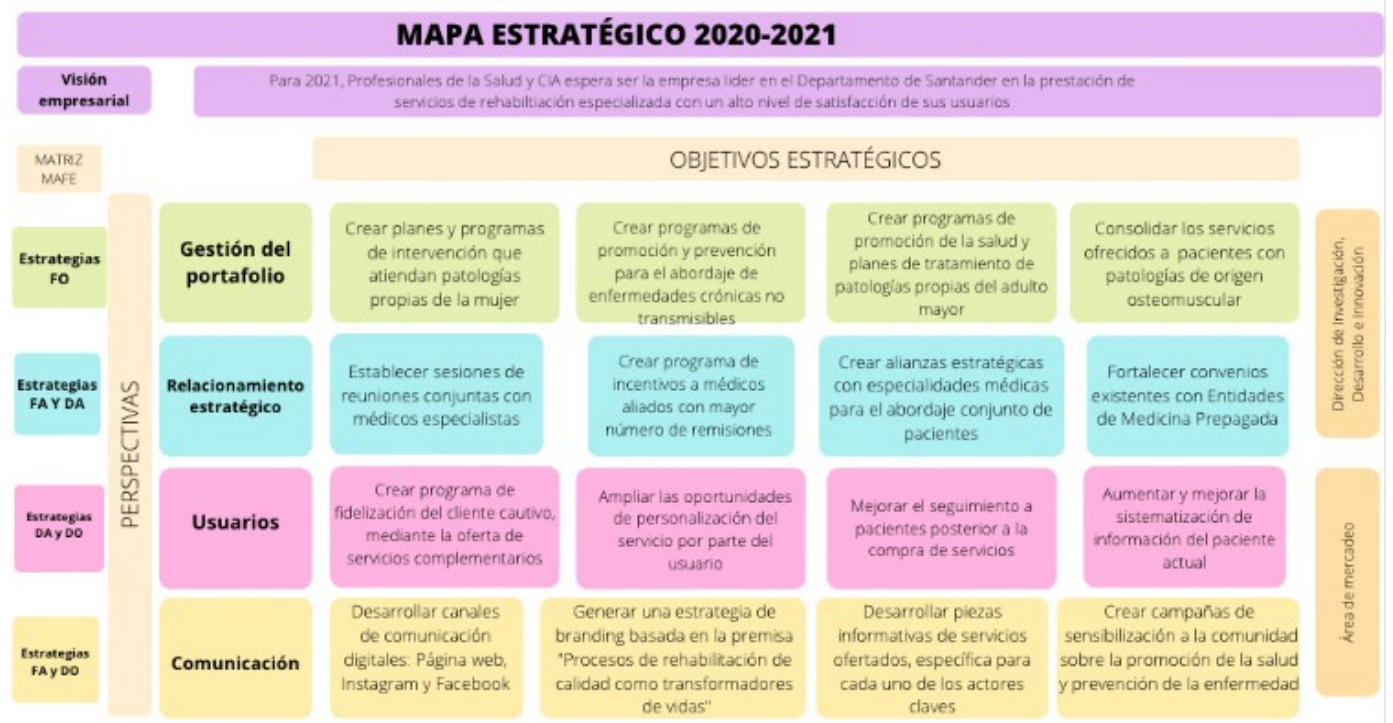

El esquema presentado en la figura 9 señala cómo la dirección de investigación, desarrollo e innovación (IDI) será, junto con la dirección general, la encargada de liderar este conjunto de estrategias. Además, se propone la creación de un área de mercadeo, que podría ir en el organigrama subordinada a la dirección de IDI, con el fin de que esta se encargue del desarrollo de actividades de fuerza de ventas y comunicación, para que estas no sean vistas como actividades con poco impacto.

\section{Conclusiones}

Estudios de investigación que permitan conocer profundamente el mercado dentro de las empresas de salud tienen gran relevancia, dado que permiten ofrecer un servicio enfocado a las necesidades y generar planes estratégicos coherentes y costos efectivos.

Esta investigación permitió dar cumplimiento a los objetivos planteados, esto, gracias al desarrollo de un análisis poblacional, con base en fuentes de información primarias y secundarias, que hizo posible la elaboración de perfiles de clientes y usuarios. Asimismo, se realizó una evaluación contextual que evidenció las necesidades del entorno y el mercado actual de la institución.

De otra parte, el diseño y la aplicación de tres instrumentos de evaluación permitieron medir las expectativas y percepciones del cliente interno y externo de la empresa. Finalmente, el análisis de la cascada de pacientes llevó a analizar e interpretar las dimensiones y brechas existentes en el sistema. Todo lo anterior permitió la generación de una segmentación de los mercados objetivos que, junto con las brechas y dimensiones establecidas, condujo a la creación de un mapa estratégico para la institución. 
De igual forma, el presente trabajo permitió conocer a profundidad el contexto organizacional de la institución objeto de estudio, a partir del cual se desarrolló un diagnóstico estratégico que, junto con las metodologías mencionadas anteriormente, sirvió para robustecer la propuesta de intervención planteada y armonizarla con el sistema de gestión de la institución.

La realización de este estudio de investigación permitirá a la gerencia general la toma de decisiones basadas en la evidencia. En este orden de ideas, el plan estratégico planteado involucrará a diversas áreas de la organización para generar una propuesta de mejoras en marketing, con el fin de aumentar el mercado potencial de la institución.

Finalmente, si bien esta investigación presenta los resultados propios de un proceso de análisis de una empresa específica, su metodología podrá ser usada para diferentes organizaciones del área de la salud.

A lo largo del proceso investigativo se encontraron diferentes limitaciones que afectaron, en cierta medida, las etapas de desarrollo del proyecto. Por ejemplo, en el proceso de búsqueda de información para la realización de la estrategia de cascada de pacientes, no se encontró evidencia del indicador 'Extensión de uso' desagregado para servicios de rehabilitación exclusivamente, por lo que el cálculo puede llegar a presentar sesgos. Sin embargo, esto no impidió la estimación del eslabón en la cascada de pacientes.

Se presentaron también algunas incoherencias en la información recolectada de diferentes fuentes primarias de carácter gubernamental, específicamente en los datos entregados por el Departamento Administrativo Nacional de Estadística (DANE) y los recibidos de parte del Ministerio de Salud y Protección Social, lo que impuso una limitante a la estimación real de la cascada de pacientes.

Finalmente, no se pudo alcanzar la muestra esperada para médicos, debido a la dificultad para poder contactarlos y a algunos inconvenientes en el reenvío del material diligenciado, a lo que se sumó la coyuntura de la COVID-19, que limitó, aún más, la posibilidad de acceder a esta población. No obstante, los resultados obtenidos permitieron establecer hechos contundentes para el desarrollo del análisis.

\section{Referencias}

Acevedo, J. (2015). Evaluación de la satisfacción en el servicio de los usuarios de la IPS servicios integrales de salud "VAQNAR" ubicada en la ciudad de Tunja Boyacá [Tesis de pregrado, Universidad Pedagógica y Tecnológica de Colombia].

Asociación Colombiana de Empresas de Medicina Integral. (2017). Cifras e indicadores del sistema de salud. Asociación Colombiana de Empresas de Medicina Integral. https://www.acemi.org.co/images/publicaciones/documentos_de_interes/documento s-interes/Informe_Cifras_2017.pdf 
Asociación Colombiana de Hospitales y Clínicas. (2018). La realidad financiera de los hospitales y clínicas. http://achc.org.co/wp-content/uploads/2018/01/RealidadFinanciera-Hospitales.pdf

Assefzadeh, S. (1996). Patient flow analysis in a children's clinic. EMHJ - Eastern Mediterranean Health Journal, 2(3), 412-417.

Brandenburger, A. (2019). Are Your Company's Strengths Really Waknesses? Harvard Business Review. https://hbr.org/2019/08/are-your-companys-strengths-reallyweaknesses

Cabello, E. y Chirinos, J. (2012). Validación y aplicabilidad de encuestas SERVQUAL modificadas para medir la satisfacción de usuarios externos en servicios de salud. Revista Medica Herediana, 23(2), 88-95.

Calixto-Olalde, M., Sawada, N., Hayashida, M., Costa, I., Trevizan, M. y Godoy, S. (2011). SERVQUAL scale: validation in the Mexican population. Texto \& Contexto Enfermagem, 20(3), 526-33.

Dixon, C., Punguyire, D., Mahabee-Gittens, M., Ho, M. y Lindsell, C. (2015). Patient Flow Analysis in Resource-Limited Settings: A Practical Tutorial and Case Study. Global Health: Science and Practice, 3(1), 126-134.

Icontec. (2008). Norma Técnica Colombiana, NTC 5801. Instituto Colombiano de Normas Técnicas y Certificación (Icontec).

Matsumoto, R. (2014). Desarrollo del Modelo Servqual para la medición de la calidad del servicio en la empresa de publicidad Ayuda Experto. Perspectivas, (34), 181-209.

Molina, G. y Vargas-Jaramillo, J. (2010). Características de la contratación entre aseguradores y prestadores de servicios de salud. Revista Gerencia y Politicas de Salud, 9(18), 110-115.

Numpaque-Pacabaque, A. y Rocha-Buelvas, A. (2016). Modelos SERVQUAL y SERVQHOS para la evaluación de calidad de los servicios de salud. Revista de la Facultad de Medicina, 64(4), 715-720.

Pérez, M., Orlandoni, G., Ramoni, J. y Valbuena, M. (2018). Percepción de la calidad en la prestación de servicios de salud con un enfoque seis sigma. Revista Cubana de Salud Pública, 44(22), 325-343.

Porter, M. (2008). The Five Competitive Forces that Shapes Strategy. Harvard Business Review, 86(1), 78-93. 
Prieto, M., Escudero, M., Suess, A., March, J., Ruiz, A. y Danet, A. (2011). Análisis de calidad percibida y expectativas de pacientes en el proceso asistencial de diálisis. Anales del Sistema Sanitario de Navarra, 34(1), 21-31.

Thompson, A. y Strikland, K. (1998). Dirección y Administración estratégicas. Conceptos, casos y lecturas. MacGraw Hill Interamericana.

Yepes, M., Ricaurte, M. y Jurado, D. (2018). Calidad percibida de la atención en salud en una red pública del municipio de Pasto, Colombia. Universidad y Salud, 20(2), 97-110.

\section{Bibliografía consultada}

Aghamolaei, T., Eghbal, T., Rafati, S., Kahnouji, K., Ahangari, S., Shahrzad, M., Kahnouji, A., Hoseini, S. (2014). Service quality assessment of a referral hospital in Southern Iran with SERVQUAL technique: patients' perspective. BMC Health Services Research, 14(322), 1-5.

Alcaldía de Bucaramanga. (2017). Análisis de situación de salud con el modelo de los determinantes sociales de salud. http://observatorio.bucaramanga.gov.co/wpcontent/uploads/2017/09/An\%C3\%A1lisis-de-Situaci\%C3\%B3n-de-Salud-con-elModelo-de-los-Determinantes-Sociales-De-Salud-2017-15abril2018.pdf

Alcaldía de Bucaramanga. (2019). Datos Abiertos. Alcaldía de Bucaramanga. http://observatorio.bucaramanga.gov.co/index.php/datos-abiertos/

Allianz. (2020). Centros médicos. Allianz. https://www.allia2net.com.co/drhc02/CO_menu/control.do

Baum, N. y Henkel, G. (2010). Marketing Your Clinical Practice: Ethically, Effectively, Economically ( $4^{\mathrm{a}}$ Ed.). Jones \& Bartlett Publishers.

Buckley, P. (2009). The Complete Guide to Hospital Marketing (2 ${ }^{\mathrm{a}}$ Ed.). HealthLeaders Media.

Colsanitas. (2020). Directorio médico. Colsanitas. https://www.colsanitas.com/usuarios/web/guest/directoriomedico?p_auth=e6MZaRcr\&p_p_id=directoriomedico_WAR_DirectorioMedicopor tlet $\& p \_p \_l i f e c y c l e=1 \& p \_p \_s t a t e=$ normal $\& p \_p \_$mode $=$view $\& p \_p \_c o l \_i d=$ column$1 \& \mathrm{p} \_$__col_count $=2 \&$ _directoriomedico_WAR_DirectorioMedicopo

DeCoursin, C., Naftalis, R. y Zippi, P. (2014). Referral Strategies for Engaging Physicians. https://na.eventscloud.com/file_uploads/f2acb119c3a175d124faf7a6648af2f2_Bayl or-DeCoursinNaftalisZippi.pdf 
Departamento Administrativo Nacional de Estadística (DANE). (2019). Censo nacional de población y vivienda 2018. https://sitios.dane.gov.co/cnpv/\#!/

Esquiaqui, R. (2016). Análisis de situación de salud con el modelo de los determinantes sociales de salud. Alcaldía de Bucaramanga. http://www.bucaramanga.gov.co/elatril/download/transparencia/ASIS-BUCARAMANGA-2015.pdf

Hernandez, R., Fernandez, C. y Baptista, P. (2010). Metodología de la Investigacióm. McGraw Hill.

Icontec. (2020). Portafolio de servicios. Icontec. https://www.icontec.org/portafoliocertificacion-sistema/

Medplus Medicina Prepagada. (2020). Especialidad. Medplus Medicina Prepagada. http://directorio.medplus.com.co/Directorio/Especialidades\#tableResultadosEspecia lidades

Ministerio de Salud y Protección Social. (2019). Caracterización de la población afiliada a las Entidades Administradoras de Planes de Beneficios (EAPB). Minsalud. https://www.minsalud.gov.co/salud/publica/epidemiologia/Paginas/caracterizacionde-la-poblacion-afiliada-a-las-EAPB.aspx

Ministerio de Salud. (2016). Gestión del mejoramiento continuo de la calidad. Orientaciones técnicas para la gestión de los resultados del monitoreo de la calidad. Imprenta Nacional de Colombia.

Physician Practice Resource Center. (2020). Medical Practice Marketing. http://www.massmed.org/physicians/practice-management/medical-practicemarketing-(pdf)/

Ponce, H. (2007). La matriz foda: alternativa de diagnóstico y determinación de estrategias de intervención en diversas organizaciones. Enseñanza e Investigación en Psicología, 12(1), 113-130.

Profesionales de la Salud y Cía. Ltda. (2019). D-GTH-O1 Manual de Perfiles y Funciones. Profesionales de la Salud y Cia Ltda.

Profesionales de la Salud y Cía. Ltda. (2020). D-GG-10 Capacidad Instalada. Profesionales de la Salud y Cía. Ltda.

Profesionales de la Salud y Cía. Ltda. (2020). Manual Integrado de Gestión. Profesionales de la Salud y Cía. Ltda. 
Supersalud. (2019). Indicadores de afiliaciones. Supersalud.

https://www.supersalud.gov.co/esco/Paginas/Delegada\%20Supervisi\%C3\%B3n\%20Institucional/Indicadores-deAfiliaciones.aspx

Sura. (2020). Directorio médico. EPS Sura. https://www.epssura.com/index.php/pacdirectorio

The W. Edwards Deming Institute. (2020). PDSA Cycle. The W. Edwards Deming Institute. https://deming.org/explore/p-d-s-a

Vargas, I. (2009). Barreras en el acceso a la atención en salud en modelos de competencia gestionada: un estudio de caso en Colombia [Tesis doctoral, Universitat Autònoma de Barcelona].

Vazquez, M., Vargas, I., Mogollon, A., Ferreira, M., Unger, J. y Paepe, P (Eds.). (2017). Redes integradas de servicios de salud en Colombia y Brasil. Un estudio de casos. Editorial Universidad del Rosario.

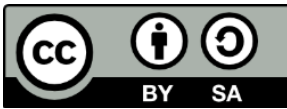

